ORIGINAL ARTICLE

\title{
Alteration in Lipid Profile Levels in Women with Subclinical
}

\section{Hypothyroidism}

\author{
S Ferduosi ${ }^{1}$ R Haque ${ }^{2}$, N Hoque ${ }^{3}$, MM Rahman $^{4}$, MH Rahman ${ }^{1}$ \\ ${ }^{1}$ Dept of Biochemistry, Dhaka Medical College, Dhaka; ${ }^{2}$ Dept of Biochemistry, \\ Sir Salimullah Medical College.Dhaka; ${ }^{3}$ Det of Biochemistry, Faridpur Medical College. Faridpur \\ ${ }^{4}$ Department of Surgery, Shahid Samsuddin Ahamed Hospital, Sylhet
}

\begin{abstract}
Thyroid hormones play an important role in regulating energy balance and metabolism of glucose and lipids. A relationship between dyslipidemia and atherosclerosis is well established in clinical hypothyroidism. Whether subclinical hypothyroidism $(\mathrm{SCH})$ is associated with lipid profile alteration, is the main concept behind the study. SCH is defined as an elevated thyroid stimulating hormone (TSH $>5 \mathrm{mIU} / \mathrm{l}$ ) and normal free thyroxine level (FT4 9.5- $25.0 \mathrm{pmol} / \mathrm{l}$ ). It is highly prevalent in elderly subjects, especially in women but it is frequently overlooked. We examined 40 women with SCH and 50 healthy controls (TSH 0.5-5.0 mIU/l and FT4 9.5$25.0 \mathrm{pmol} / \mathrm{l})$. None of the patients had been previously treated with thyroxine. In all participants we measured blood pressure, BMI, TSH, FT4 and fasting serum lipid profile. We conclude that $\mathrm{SCH}$ in middle aged women is associated with hypertension and significant increase of TC $(\mathrm{p}<0.001)$, LDL-C $(\mathrm{p}<0.001)$, TG $(\mathrm{p}<0.001)$ and decreased level of HDL$\mathrm{C} \mathrm{p}<0.001)$ in comparison to euthyroid Controls. SCH patients showed significant positive correlation between TSH and total cholesterol $(\mathrm{r}=.492, \mathrm{p}<0.01)$, LDL-C $(\mathrm{r}=.355, \mathrm{p}<0.05)$ and TG $(r=.274, p<0.05)$ and negative but nonsignificant corelationship with HDL-C $(r=-$ $.058, \mathrm{p}=0.361$ ). Dyslipidemia is one of the established risk factors of cardiovascular diseases. Therefore, this study indicates that monitoring of lipid level in $\mathrm{SCH}$ patients would be helpful in preventing cardiovascular diseases.
\end{abstract}

Key Words: Lipid profile, SCH, Euthyroid

\section{Introduction}

Thyroid disorder is a common endocrine disorder affecting about 300 million people worldwide and over half are presumed to be unaware of their condition ${ }^{1}$. Thyroid diseases are primary conditions that affect the amount of thyroid hormone being produced. Excess production leads to hyperthyroidism while diminished production leads to hypothyroidism. Hypothyroidism and hyperthyroidism are two widespread thyroid problems of which hypothyroidism is much more common. These disorders are eight times more common in

Bangladesh J Med Biochem 2015; 8(1): 10-15 women than in men ${ }^{2}$. Thyroid hormones are important modulators of intermediary metabolism. They have significant effect on the synthesis, mobilization and metabolism of lipids, although degradation is influenced more than synthesis. Consequently, thyroid dysfunction particularly hypothyroidism is associated with dyslipidemia which increases the risk of endothelial dysfunction, hypertension and cardiovascular diseases ${ }^{3}$. It is universally accepted that there is a correlation of lipid profile alteration among hypothyroid patients 
Alteration in Lipid Profile Levels in Women with Subclinical Hypothyroidism and since half century ago, there were documented studies, on whether the dyslipidemia finally leads to cardiovascular diseases $^{4}$. Overt hypothyroidism may associate with increased risk for cardiovascular diseases (CVDs) as indicated by hypertension, hypercholesterolemia and increased low-density lipoprotein cholesterol (LDL-C) level. However not all patients have hypertension or abnormal lipid profiles, suggesting that other factors may be involved as risk factor for $\mathrm{CVDs}^{5}$.

$\mathrm{SCH}$ is defined as an asymptomatic condition with small elevation of serum thyroidstimulating hormone (TSH) levels and normal free thyroid hormone 6 . It is referred to as a state of mild thyroid failure and is essentially a laboratory diagnosis ${ }^{7}$. Such subjects eventually will lead to have lower metabolic rate and clinical manifestation such as overweight, fatigue, hypertension and depression ${ }^{8}$. This condition is more common in the elderly and is found twice as often in women as in men ${ }^{9}$. Uzunlulu et al. noted that only female gender was associated with the presence of $\mathrm{SCH}^{10}$.

$\mathrm{SCH}$ may be associated with increased risk of coronary artery disease (CAD), peripheral vascular disease and various biochemical abnormalities including increased LDL-C level, increased total cholesterol and serum triglyceride values ${ }^{7}$. Moreover, it is likely to be a risk factor for atherosclerosis and coronary disease $e^{3,5.7}$. However the controversy persists regarding the lipid profile alterations in $\mathrm{SCH}$ and its clinical significance. Some case-control studies, have reported increased levels of serum total cholesterol, triglycerides and LDL-C in subjects with SCH compared with euthyroid controls $3,6,8,11,12$. Several large cross-sectional studies found no significant differences in total cholesterol, TG or LDL-C between $\mathrm{SCH}$ subjects and euthyroid controls $5,7,9$.

Diseases of thyroid gland are amongst the most abundant endocrine disorder in the world second only to diabetes mellitus ${ }^{1}$. SCH is much more common than overt hypothyroidism ${ }^{7}$. Therefore early diagnosis and treatment may prevent the onset of overt hypothyroidism and its associated effects. At present, endocrine diseases are increasing worldwide. Hypothyroidism is the most common thyroid disorder in Bangladesh according to different studies on thyroid disorders in the 'Endocrine Clinic of BSMMU14. Frequency of $\mathrm{SCH}$ is also documented to be high in Bangladesh ranging from $4 \%$ to $8.5 \% 15$ and the main bulk comprises the female ${ }^{16}$.

The aim of our study was to estimate serum lipid profile in women with $\mathrm{SCH}$ and the relationship between them as there is no such study related to lipid profile changes among these women in Bangladesh.

\section{Materials and Methods}

The present cross sectional study was conducted on 90 women in the age range of 20-60 years, visiting the Nuclear Medicine Centre of Dhaka Medical College from July 2010 to May 2011 with suspicion of thyroid disorders. A detailed history with emphasis on symptoms related to impaired thyroid function was recorded. Subclinical hypothyroidism was established on the basis of elevated TSH level $(>5 \mathrm{mlU} / \mathrm{l})$ and normal FT4 (9.5-25.0 pmol/1) level, while the patients suffering from overt hypothyro oidism (primary/secondary), undergoing treatment with thyroxine/antithyroid drugs, diabetes mellitus, cardiac or renal diseases, undergoing treatment with antilipidemic drugs, pregnant women and women on oral contraceptives were excluded. Women having normal serum TSH (0.5-5.0 mIU/l) and normal FT4 level were taken as euthyroid controls. The study was carried out in Department of Biochemistry, BSMMU, Dhaka. The research protocol was approved by the Institutional Ethical Committee and informed writen consents were obtained from all the participants.

Blood Pressure was measured twice following standard procedure in sitting position and mean 
pressure was taken as blood pressure. Height was measured in $\mathrm{cm}$ and weight was measured in $\mathrm{kg}$ with light clothings ${ }^{1}$ and without footwear. Blood sample was collected in the morning at least after 12-hour overnight fasting. Data was collected in a preformed data collection sheet. For biochemical investigation serum was separated by centrifugation at $3000 \mathrm{rpm}$ for 5 minutes and was stored at $4{ }^{\circ} \mathrm{C}$ until analysis. TSH, FT4, TC, HDL-C and TG were estimated according to the protocol mentioned in the test kits from HUMAN, Germany.

The normal reference ranges according to the kits are: TSH (0.5-5.0 mIU/L), FT4 (9.5-25.0 $\mathrm{pmol} / \mathrm{L})$. Normal values for lipid profile parameters are total cholesterol (150-220 $\mathrm{mg} / \mathrm{dl})$, triglyceride $(100-150 \mathrm{mg} / \mathrm{dl})$, HDL-C $(>50 \mathrm{mg} / \mathrm{dl})$, LDL-C $(<-130 \mathrm{mg} / \mathrm{dl})^{8}$.

\section{Statistical Analysis}

Data were analyzed using software program SPSS (Statisical Package for Social Sciences) for Windows version 19.0. The result was expressed as mean \pm SD. The statistical difference was evaluated by Students't' test and Pearson correlation coefficient test at $\mathrm{p}<0.05$. was considered statistically significant.

\section{Results}

A total 90 subjects were enrolled for the study. All were females. They were classified into Euthyroid and Subclinical hypothyroid $(\mathrm{SCH})$ according to their serum TSH and FT4 status. Table I shows the distribution of the study subjects according to age. Majority of the study subjects in the Euthyroid group and Hypothyroid group we are in the age group 3140 yrs. Mean age for euthyroid and SCH was $37.72 \pm 10.33$ yrs and $36.85 \pm 10.38$ yrs respectively.
Table I: Distribution of euthyroid and $\mathrm{SCH}$ patients on the basis of age

\begin{tabular}{lcc}
\hline Age $($ years) & Euthyroid $(\mathbf{n}=\mathbf{4 0})$ & $\mathbf{S C H}(\mathbf{n}=\mathbf{5 0})$ \\
\hline $20-30$ & 15 & 10 \\
$30-40$ & 20 & 16 \\
$40-50$ & 10 & 11 \\
$50-60$ & 5 & 3 \\
Total & 50 & 40 \\
\hline
\end{tabular}

Table II shows the comparison of BMI, systolic bloods pressure (SBP), diastolic blood pressure (DBP) and serum lipid profile parameters between euthyroid and $\mathrm{SCH}$ subjects by unpaired ' $t$ ' test. SBP, DBP, serum triglyceride, serum total cholesterol, LDL- cholesterol were found significantly higher in the $\mathrm{SCH}$ group compared to the Euthyroid group. On the other hand HDL-C was significantly higher in the Euthyroid group.

Table II: Comparison of BMI, blood pressure, TSH, FT 4 and lipid profile parameters between euthyroid and SCH subjects

\begin{tabular}{llll}
\hline Variable & $\begin{array}{c}\text { Euthyroid(n=50) } \\
\text { meantSD }\end{array}$ & $\begin{array}{l}\text { SCH(n=40) } \\
\text { mean } \pm \text { SD }\end{array}$ & p value \\
\hline BMI (Body mass index) & $24.2 \pm 2.5$ & $28.0 \pm 2.9$ & $<0.01$ \\
SBP (Systolic blood pressure) & $116.4 \pm 9.9$ & $125.2 \pm 11.8$ & $<0.01$ \\
DBP (Diastolic blood & $76.4 \pm 6.5$ & $83.4 \pm 0.87$ & $<0.01$ \\
pressure) & & & \\
TSH & $2.53 \pm .582$ & $11.28 \pm 4.03$ & $<0.01$ \\
FT4 & $15.33 \pm 3.33$ & $13.43 \pm 2.57$ & $>0.05$ \\
Triglyceride & $118.76 \pm 34.42$ & $166.27 \pm 47.08$ & $<0.01$ \\
Total cholesterol & $175.78 \pm 22.69$ & $212.33 \pm 20.66$ & $<0.01$ \\
HDL -Cholesterol & $44.02 \pm 6.82$ & $40.53 \pm 7.19$ & $<0.01$ \\
LDL -Cholesterol & $108.56 \pm 20.92$ & $138.00 \pm 19.58$ & $<0.01$ \\
\hline
\end{tabular}


Alteration in lipid profile levels in women with subclinical

Table III: Pearson Correlation Co-efficient between TSH, FT4 and lipid profile

\begin{tabular}{|c|c|c|c|c|c|}
\hline & & $\mathrm{TC}$ & LDL - C & HDL - C & T G \\
\hline \multirow[t]{2}{*}{$\mathrm{SCH}$} & TSH & ** .492(.001) & 1) $.355(.012)$ & $-.058(.361)$ & $* .274 \quad(.043)$ \\
\hline & FT4 & $.202(.105)$ & $.245(.064)$ & $.024(.442)$ & $-.137(.200)$ \\
\hline
\end{tabular}

**Correlation is significant at 0.01 levels (1-tailed) *Correlation is significant at 0.05 level (1-tailed)

Subclinical hypothyroid patients showed significant positive correlation between TSH and total cholesterol $(\mathrm{r}=0.492)$, triglycerides $(\mathrm{r}=0.274)$ and LDL-C $(\mathrm{r}=0.355)$, negative corelationship with HDL-C $(r=-0.058)$.

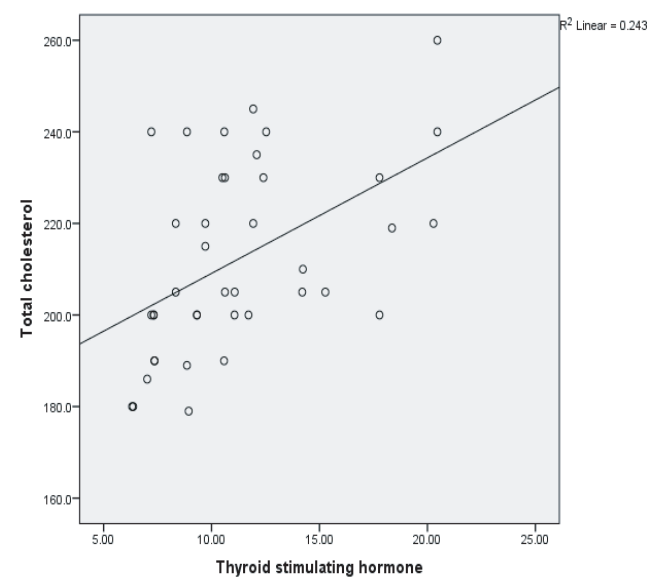

Fig-1: Correlation between TSH and total cholesterol

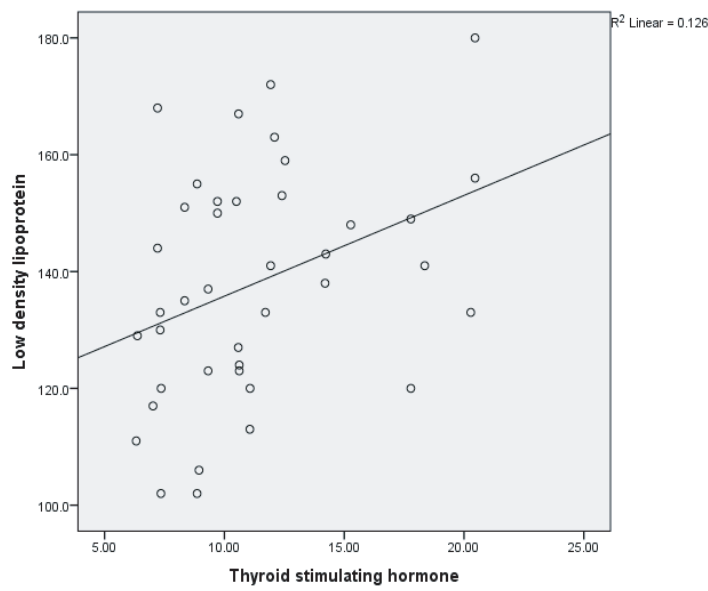

Fig 2: Correlation between TSH and serum LDL-C

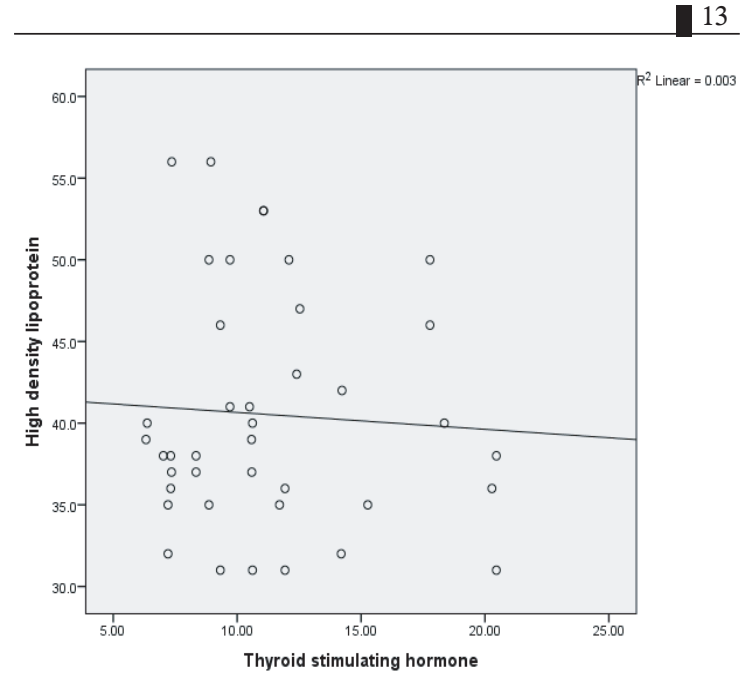

Fig 3: Correlation between TSH and serum HDL-C

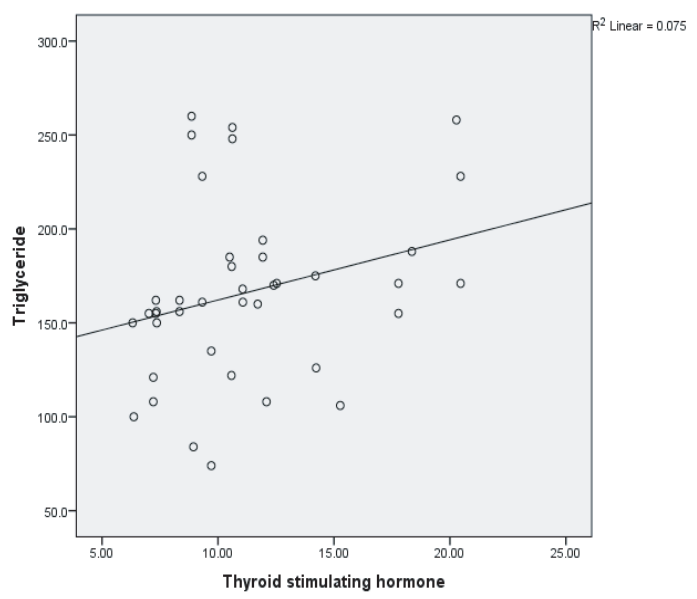

Fig 4: Correlation between TSH and serum TG

\section{Discussion}

Hypothyroidism is a progressive disorder presenting with different degrees of thyroid failure and metabolic consequences. The correlation between lipid profile changes and overt hypothyroidism is well established. However, lipid profile alterations in $\mathrm{SCH}$ are controversial; some reports showing positive correlation and prompt reversal of changes following treatment ${ }^{7}$ and few studies refuting any correlation between the two $0^{5,9}$. In this study 40 newly diagnosed women of 
subclinical hypothyroidism and 50 women of euthyroid control were enrolled. The prevalence of hypothyroidism was found higher in the age group 30-50 years which is in accordance with the findings by Regmi et al. ${ }^{3}$ while Huesten et al. ${ }^{9}$ noted that, by the age of 65 years the overall prevalence of the disorder is more among in females than among males. This may be either due to lesser number of elderly patients being referred for the test or the symptoms of hypothyroidism are often confused with normal ageing process and coexisting diseases which may result in greater number of elderly patients being undiagnosed. Mean values of systolic and diastolic blood pressure were found higher in SCH patients versus controls in our study which corresponds to the findings of a study done by Uzunlulu et al. ${ }^{10}$ But Luboshitzky et al. ${ }^{5}$ reported only increased mean systolic blood pressure in $\mathrm{SCH}$ cases.

In this study, all the parameters of lipid profile i.e., TC, LDL-C, TG except HDL-C were found increased in $\mathrm{SCH}$, while concentration of HDL-C was decreased in subjects with $\mathrm{SCH}$ as compared to euthyroid Controls and the differences were statistically significant. Many researchers found higher and significant level of TC, LDL-C and TG level compared to euthyroid controls $3,6,8,11,12$. But few authors found no significant difference in lipid profile between SCH patients and euthyroid Controls ${ }^{7,9}$.

It is well known that thyroid hormone has pervasive effects on the transport of the plasma lipoproteins. Cholesterol and LDL-C typically accumulate in the plasma of patients with hypothyroidism because thyroid hormone stimulates LDL-C receptor activity, resulting in decreased catabolism of LDL-C and IDL $^{6,17}$. According to Duntas ${ }^{18}$ increase of TC and LDL-C can be attributed to the effect of thyroid hormone on expression of LDL receptors and CYP7A, a rate limiting enzyme in bile acid synthesis. Decreased thyroid function not only increases the number of LDL particles but also promote LDL oxidation, thereby increasing the risk of atherosclerosis ${ }^{3}$.
S Ferduosi, R Haque, N Hoque el al

A significant increase in serum triglycerides level in our study is in agreement with the reports by Singh et al. ${ }^{7}$ and Regmi et al. ${ }^{3}$ This increase may be due to decreased activity of lipoprotein lipase that is responsible for the clearance of TG rich lipoproteins ${ }^{3}$ or decrease in plasma post heparin lipolytic activity in the hypothyroid state accounting for raised TG level ${ }^{19}$. But Waterhouse et al. ${ }^{20}$ found impaired level of TG, but unchanged level of TG was also reported. ${ }^{6}$ The mean fasting HDL-C was significantly lower in $\mathrm{SCH}$ group compared to euthyroid controls, which is supported by some authors 7,11 , while Regmi et al. ${ }^{3}$ found increased level of HDL-C in SCH patients. In some previous studies, HDL-C has been reported to be unchanged among SCH patients ${ }^{5,6,9}$. There may be involvement of dietary or physical activity factors which may be responsible for the lower values yielded in this study.

Positive correlation was observed between TSH and TC $(\mathrm{P}=.001)$, TSH and LDL-C $(\mathrm{p}=.012$ and TSH and TG $(\mathrm{p}=.043)$ and negative correlation between TSH and HDL-C $(p=.361)$. These findings are consistent with the findings of many others $6,11,12$. Non significant relationship was observed between FT4 and lipid parameters in $\mathrm{SCH}$ patients.

\section{Conclusion}

It may be concluded that $\mathrm{SCH}$ is associated with an atherogenic lipid and lipoprotein profile, characterized by an increased concentration of TC, LDL-C and TG and by decreased HDL-C level. This may accelerate the risk of atherosclerosis and coronary artery diseases in patients with $\mathrm{SCH}$. It was also observed that the abnormal lipid pattern is fully reversed to normal by treatment with thyroxine. So, monitoring of lipid profile in patients of $\mathrm{SCH}$ is necessary along with substitution therapy to prevent the cardiovascular risk from dyslipidemia. 


\section{References}

1. Madhukar A, Prabin G, Nirakar R, Pratibha A, Dipendra RP. A prevalence of thyroid dysfunction in Kathmandu University Hospital, Nepal. Biomed Res 2010; 21(4): 411-415.

2. Peter PAS eds. Epidemiology of Thyroid dysfunction-hypothyroidism and hyperthyroidism. Thyroid Int 2009; 2: 1-16.

3. Regmi A, Shah B, Rai BR and Pandeya A. Serum lipid profile in patients with thyroid disorders in central Nepal. Nepal Med Coll J 2010; 12(4):253256.

4. Azad RM. The state of serum lipid profiles in subclinical hypothyroidism: A review of the literature. Pak J Biol Sci 2010; 13(11):556-562.

5. Luboshitzky R, Avraham I and Paula H. Metabolic syndrome and Insulin resistance in women with subclinical hypothyroidism. The Endocrinologist 2010; 20(1):29-32.

6. Turhan S, Sezer S, Erden G, Guctekin A, Ucar F, Ginis Z, Ozturk O, Bingol S. Plasma homocysteine concentration and serum lipid profile as atherosclerotic risk factors in subclinical hypothyroidism. Ann Saudi Med 2008; 28: 96-10.

7. Kuldip S \& Saranpal S. Alterations in lipid fraction levels in subclinical hypothyroidism in North Indian population. Ind J Fundament Appl life Sci 2011; 1(2):127-132.

8. Farah AK, patil SKB , Amar ST, Mohammad FK, Murugan K. Lipid profile in thyroid dysfunction: A study on patients of Bastar. JCAM 2012; 1-3.

9. Hueston WJ, Pearson WS. Subclinical hypothyroidism and the risk of hypercho lesterolemia. Ann Fam Med 2004; 2: 351-355.
10. Uzunlulu M, Yorulmaz E and Oguz A. Prevalence of subclinical hypothyroidism in patients with metabolic syndrome. Endocr J 2007; 54(1): 71-6.

11. Shashi A, Sharma N. Lipid profile abnormalities in hypothyroidism. Int J Sc nat 2012; 3(2):353-360.

12. Pradeep S, Dibyaratna P, Sapna G, Geeta S, Pathak M.S. Hypothyroidism causing dyslipidemia in both subclinical and overt hypothyroidism. Ind $\mathbf{J}$ Basic Appl Med Res 2013; 2(7):779-788.

13. Alam MN, Haq SA, Ansari MAG, Karim MA, Das KK, Baral PK. et al. Spectrum of thyroid disorder in IPGMR, Dhaka, Bangladesh. Bang J Med 1995; 6:53-58.

14. Sutradhar SR. Approach to subclinical thyroid disease. J Bang Coll phys surg 2008; 26(2):91-96.

15. Selim S. Hypothyroidism. The Daily Sun 2011; 24:2.

16. Atthans BU, Staub JJ and De-Lechel R. LDL/HDL changes in subclinical hypothyroidism: possible risk factor for coronary heart disease. Clin Endocrinol 1988; 28:157-63.

17. Bauer DC, Ettinger B, Browner WS. Thyroid function and serum lipids in older women: A population-based study. Am J Med 1998; 104:546-551.

18. Duntas LH. Thyroid disease and lipids. Thyroid 2002; 12: 287-93.

19. Nikkila EA and Kekki M. Plasma triglyceride metabolism in thyroid disease. J Clin Invest 1972; 51(8): 2103-2144.

20. Waterhouse DF, Molaughlin AM, Walsh CD, Sheehan F, Shea DO. An examination of the relationship between normal range thyrotropin and cardiovascular risk parameters: A study in healthy women. Thyroid 2007; 17(3): 243-248. 